\title{
Streaming birefringence in extensional flow of polymer solutions
}

\author{
W. H. Talbott and J.D. Goddard
}

With 13 figures and 3 tables

\section{Introduction}

'Streaming birefringence' is the term usually employed to describe the optical birefringence induced by deformation accompanying flow in fluid-like materials. Neumann and Maxwell (1) appear to have been the first to propose a linear relation between applied stress and birefringence. Subsequent descriptions of stress-optical behavior in isotropic solids employ this linear relation almost always and with some success. In addition, many experimental measurements on polymeric fluids tend to confirm the same linear stress-optical or Maxwell relations:

$\Delta=\hat{n}_{1}-\hat{n}_{2}=C\left(\hat{\sigma}_{1}-\hat{\sigma}_{2}\right)$,

$\chi_{D}=\chi_{S}$

where $\Delta$ is the birefringence; $\hat{n}_{1}$ and $\hat{n}_{2}$ are the principal indices of refraction of the stressed material; $\hat{\sigma}_{1}$ and $\hat{\sigma}_{2}$, the principal stresses; $C$ is the stress-optical coefficient, a material constant; $\chi_{D}$ is the orientation angle of the major axis of the dielectric ellipsoid; and $\chi_{s}$ is the orientation angle of the major axis of the stress ellipsoid. The equations are appropriate to observations along one optical axis and are equivalent to Maxwell's assumption that the axes of polarization of the light within the material are parallel to the directions of the principal stresses.

Nearly all flow birefringence investigations on polymeric liquids, however, have been confined to simple shear. Rigorous verification of the linear relations in such shearing flows requires in principle four separate measurements - shear stress, primary normal stress, birefringence, and extinction angle. Direct experimental measurement of these four quantities has rarely been achieved. By contrast, simple uniaxial extension provides a desirable alternative, since the symmetry of this 530 flow requires coaxiality of the stress and dielectric tensors. Hence, only two distinct measurements are necessary and this flow affords a more direct test of the linearity of the stressoptical relations. These and other results follow from phenomenological theories of birefringence in fluids such as those presented by Rivlin and Smith (2) and Coleman, Dill, and Toupin (3). These theories also point out the highly restricted nature of the linear Maxwell relations. It is therefore worthwhile to review briefly the existing empirical or moleculartheoretic basis for the linear theory.

For simple shear, verification of the linear relations has been limited, and the necessary measurements of primary normal stress have been done only at relatively low rates of shear or over a narrow range of shear rates. Furthermore, some published data by prominent workers in the field $(4,5)$ shows the birefringence exhibiting a definite nonlinear relation to the principal stress difference, obviously contradicting [1].

The elastic liquid theory proposed by Lodge (6) provides a molecular rationale for the linear relation in concentrated polymer solutions and melts. This theory appears to have provided the impetus to the pioneering series of experiments of Philippoff and coworkers who measured the stress-optical relations for a variety of systems in shearing flow.

The results of Philippoff and coworkers $(4,7,8)$ generally support the validity of [1]. In addition, their independent primary normal-stress measurements $(4,7)$ indicate coaxiality of the stress and dielectric tensors, at least for those stress levels at which optical extinction angle and primary normal-stress measurements overlap. Solutions of nitrocellulose in n-butyl acetate, however, show clear departures from linearity at high stress levels (4). Furthermore, the extensive independent primary normal-stress measurements of Brodnyan et al. (8) do not conclusively demonstrate coaxiality of the stress and dielectric tensors, a point which has already been noted by Wayland (9).

In later work, Philippoff (10) established a method to subtract the effects of solvent viscosity and solvent birefringence from that of the solution, as a means of eliminating concentration effects in relatively dilute 
solutions. His subsequent studies on solutions of various polystyrenes in Aroclor 1248, a strongly birefringent solvent, showed linear stress-optical behavior. These studies, however, either presumed (11) the coaxiality of the stress and dielectric tensors or based their coaxiality on very limited primary normal-stress measurements (12). Philippoff showed (11) that the solvent birefringence for these solutions could be neglected at higher polymer concentrations (greater than approximately $1 \%$ by weight).

Wales (1) provides solid evidence of the constancy of the stress-optical coefficient for several polymer melts and fluids well into the range of significant nonnewtonian shear viscosity behavior of these materials. Independent measurements of primary normal stress, where available, tended to confirm the coaxiality of the stress and dielectric tensors. Gortemaker et al. (14) present similar results for a commercial polyethylene.

Wayland (9) and Harris (13), among others, stated reservations concerning the applicability of the Maxwell relations to unsteady flow. Recent investigations, which include independent determination of primary normal stress, by Janeschitz-Kriegl and coworkers $(14,15)$ on polymer melts support the validity of the linear law for these melts in transient experiments involving suddenly-imposed constant shear or sudden cessation of steady shear flow. However, one concludes that the shear rates here are relatively low in that, for the shear rates imposed in their studies, the viscosity function of the polymers does not significantly depart from the limiting newtonian value.

In contrast to the numerous studies of streaming birefringence in simple shearing flows, measurements of on-line extensional birefringence during an extensional flow are few.

Wales and Philippoff (16) have cited the studies of Ziabicki and Kedzierska (17-19) on polymer melts as evidence of the validity of the stress-optical laws in extensional flow of spun liquid filaments. Only one polymer melt (polyethylene terephthalate) of the six tested, however, produced a linear relation between their measured birefringence and their calculated stress. Moreover, the birefringence in these studies appears to have been measured after the filaments had solidified.

From among other measurements of birefringence in filaments $(20-26)$, only Hamana et al. (23), Dees and Spruiell (25), and Ishibashi and Furukawa (26) attempted to relate local birefringence to local spinline stress. Only one polymer melt (polyethylene) (23) showed linear stress-optical behavior; several materials showed limiting linear behavior at low values of spinline tensile stress $(23,26)$.

The recent results of Oda, White and Clark (27) in non-isothermal experiments, including some on-line measurements, show linear stress-optical behavior at low stress levels but exhibit departures from linearity at higher stresses. For the non-isothermal experiments, their data seem to show that comparable stress-optical behavior obtains from either on-line or quenchedsample measurements. Matsumoto and Bogue (28) measured on-line birefringence in isothermal and nonisothermal experiments in extension of polystyrene film samples. Their results indicate linear stress- optical behavior at low stress levels but pronounced departures from linearity at high stress levels.

The above studies may provide valuable information concerning the development of molecular orientation under actual processing conditions. The presence of thermal gradients and solidification processes, however, somewhat complicates interpretation of the observed stress-optical behavior. One still tends to share the conclusion of Peterlin (29), that few reliable data for birefringence in extensional flow are available, which provides much of the motivation of the work on polymer solutions to be described here.

\section{Experimental method and apparatus}

\subsection{Experimental arrangement}

A filament-spinning arrangement (fig. 1) generated the extensional flow. A pump, providing constant volumetric flow, extruded the fluid from the nozzle and a rotating draw wheel took up the resulting filament. Deflection of the nozzle at a fixed draw wheel speed provided the measurement of the (constant) force acting on the nozzle tip. Control of the speed of the draw wheel provided a means to vary the extension rates and the use of two different volumetric flow rates extended the range of the data. Photographs of the filament provided measurement of diameter versus axial position from which the local stress and kinematics were derived.

A narrow beam of polarized light passed through the extending filament and the transmitted intensity method, utilizing crossed and parallel positions of a polarizer-analyzer combination, served to measure the local birefringence at one local axial station.

The primary measurements were force on the nozzle tip, recorded as displacement of the tip; filament diameter, measured from a photograph of the filament; and transmitted light intensity, recorded as equivalent photocurrent at two different analyzer settings. Other measurements included static index of refraction, density, surface tension, volumetric flow rate, and various measurements involved in system calibrations.

\subsection{Kinematic and dynamical aspects of the extensional flow}

The tensile stress in the isothermal spinning experiment involves contributions from grav- 


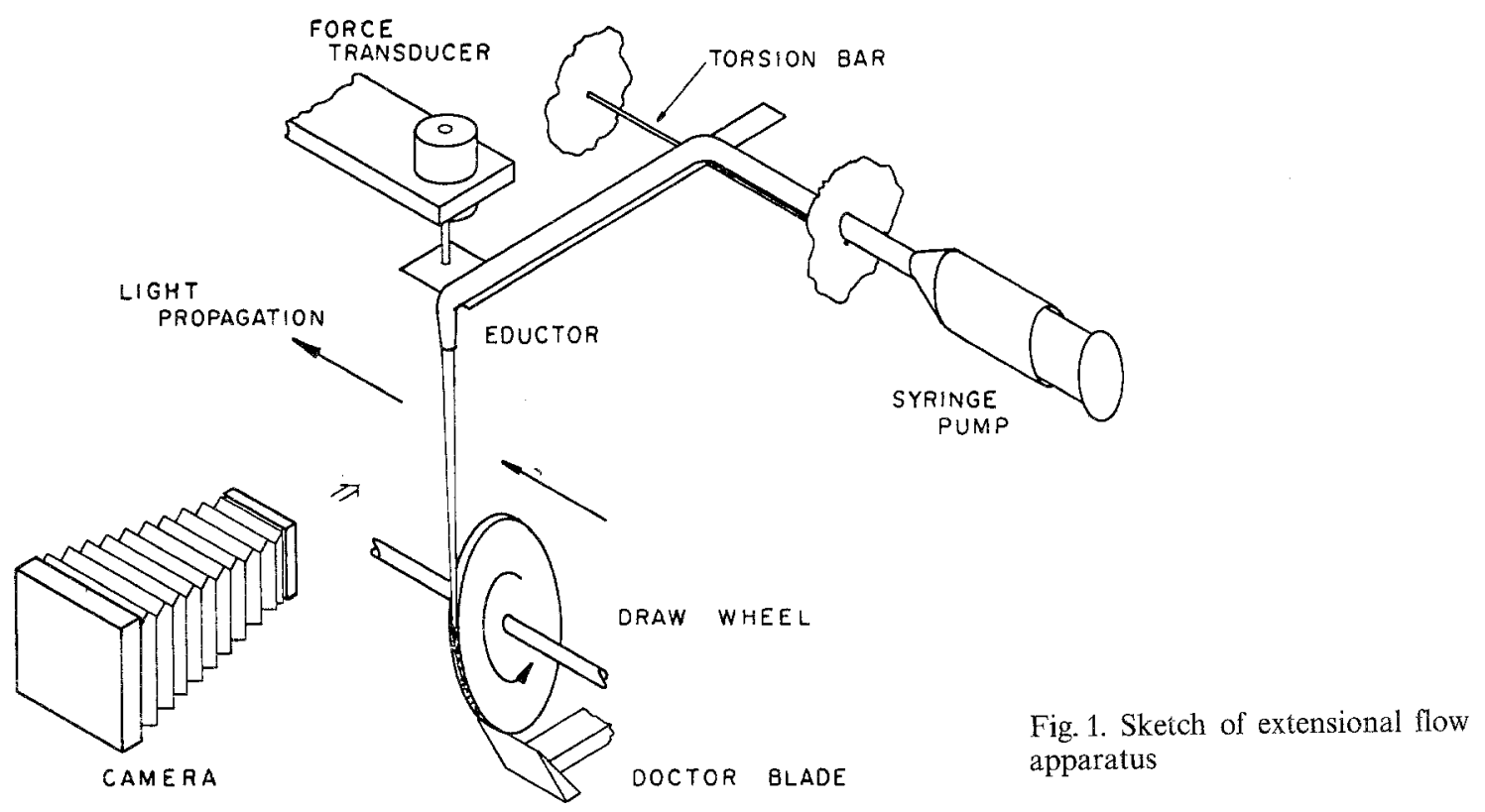

itational, inertial, surface tension, and air drag forces.

If drag forces are neglected and a one-dimensional flow is assumed, the the local axial or spinline stress for the constant force spinning experiment is given by

$\tau=c v+\rho v \int_{0}^{z}\left\{-g+\left[v+\frac{\sigma}{\rho} \sqrt{\frac{\pi}{Q v}}\right] \frac{d v}{d z}\right\}$

$$
\cdot d(\ln v)
$$

where

$c=\tau(0) / v(0)=F_{0} / Q$

and $F_{0}$ is the tensile force on the nozzle tip and $v$ is the filament velocity. For experiments performed at constant volumetric flow rate $Q$, measurements of the filament diameter, combined with the equation of continuity, permit the numerical integration of [2]. For all the work discussed here, the point of maximum filament diameter is taken to be $z=0.0$. Weinberger and others discuss this procedure and [2] more fully elsewhere.

\subsection{Optical equations for the extensional flow field}

The section of the thin filament situated between the polarizer and the analyzer can be treated as an optical retarder. Then, the light intensity transmitted by the analyzer is given by

$I_{1}=I_{0} \sin ^{2}\left(\frac{\delta}{2}\right)$

Table 1. Systems investigated

\begin{tabular}{lllll}
\hline Polymer & $\begin{array}{l}\text { Polymer } \\
\text { characterization }\end{array}$ & Supplier & Solvent & $\begin{array}{l}\text { Polymer concentra- } \\
\text { tion in solvent }\end{array}$ \\
\hline Separan AP 30 & $\begin{array}{l}\text { Polyacrylamide, } \\
M W, 2-3 \cdot 10^{6}\end{array}$ & Dow & $1: 1$ mixture (weight) & $1 \frac{1}{2} \%$ (weight) \\
& glycerol-water & $2 \frac{1}{2} \%$ (weight) \\
Separan AP 273 & Polyacrylamide, & Dow & $1: 1$ mixture (weight) & $1 \frac{1}{2} \%$ (weight) \\
& $M W,>3 \cdot 10^{6}$ & & glycerol-water & $2 \frac{1}{2} \%$ (weight) \\
Styron 686 & Polystyrene ${ }^{1}$ ) & Dow & Monsanto Aroclor & $5 \%$ (weight) \\
& $\bar{M}_{n}=95890$ & & $1254^{2}$ ) & \\
& $\bar{M}_{w}=270924$ & & &
\end{tabular}

1) Samples and analyses by courtesy of Dr. Henry J. Karam, Dow Chemical, USA.

$\left.{ }^{2}\right)$ Sample by courtesy of Mr.W. B. Papageorge, Monsanto Industrial Chemicals Co. 


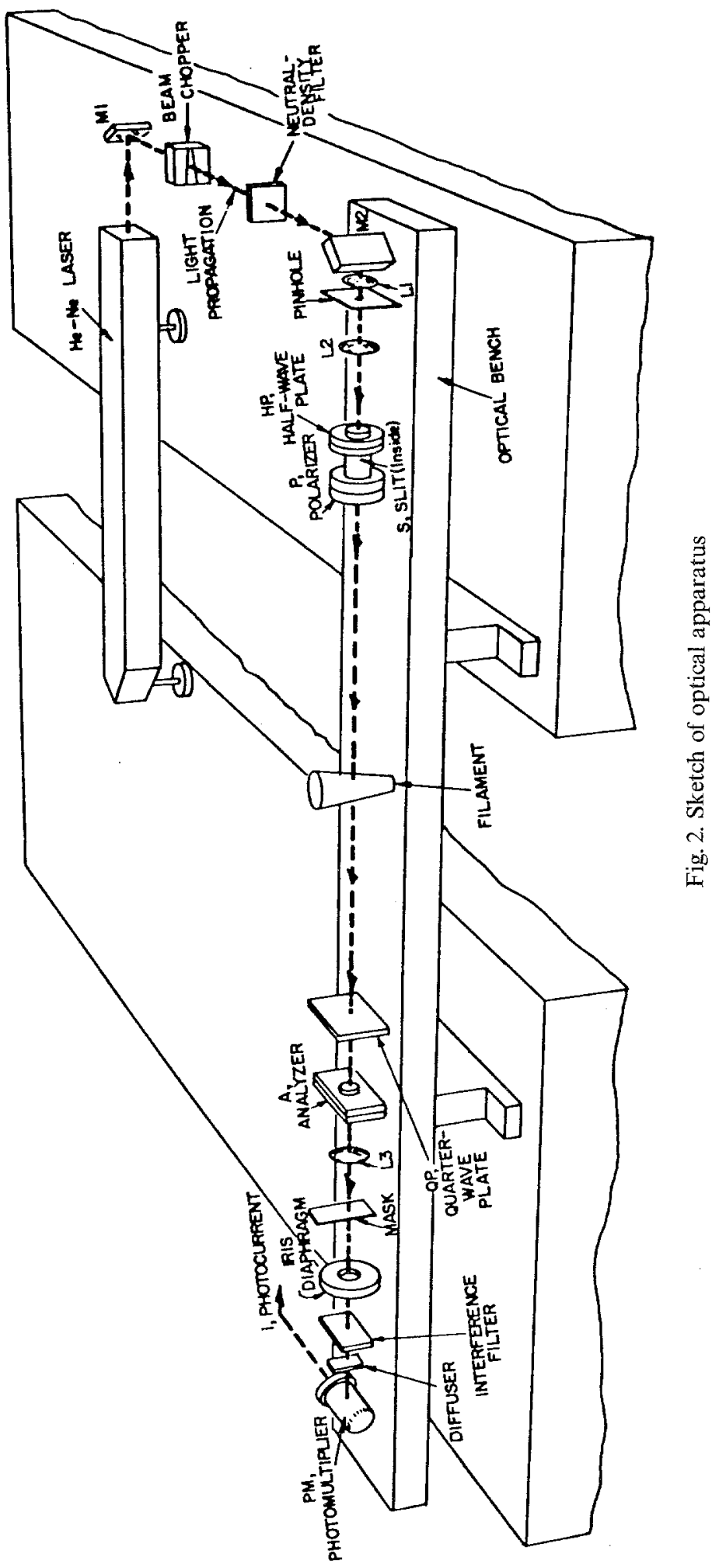


for the polarizer-analyzer in the crossed position and

$I_{1}=I_{0}\left\{1-\sin ^{2}\left(\frac{\delta}{2}\right)\right\}$

for the polarizer-analyzer in the parallel position. In [4] and [5], $I_{0}$ is the effective incident light intensity and

$\delta=\delta_{z z}-\delta_{r r}=\frac{2 \pi d}{\lambda}\left(n_{z z}-n_{r r}\right)=\frac{2 \pi d}{\lambda} \Delta$

where $\Delta$ is the local birefringence in the filament; $d$ is the local filament diameter; and $\lambda$ is the wavelength of light used in the experiment. Eqs. [4] and [5], which represent the transmitted light intensity for the azimuth of the polarizer aligned at $45^{\circ}$ with respect to the axis of extension, allow for elimination of the incident intensity $I_{0}$.

\subsection{Experimental fluids}

Our experiments studied five distinct fluids, including two polyacrylamides of different molecular weight dissolved in a 1:1 mixture of water and glycerol at two different concentrations each, and a $5 \%$ solution of a commercial polystyrene in Aroclor 1254. Table 1 summarizes the properties of these solutions. - See Talbott (31) for a discussion of the preparation of these solutions.

\subsection{Details of the apparatus}

The extensional flow device, illustrated in figure 1 , was similar to that used by Weinberger $(30,32)$.

A syringe pump capable of two constant volumetric flow rates fed the fluid to the nozzle. The draw wheel had an angular velocity range of 0.018 to $0.30 \mathrm{rad} / \mathrm{sec}$.

A view camera photographed the filament using Polaroid Type 105 positive/negative film. Nominal magnification of the filament recorded on the film was $2: 1$. A recording microdensitometer measured the filament diameters directly from the negative. This instrument scanned each negative at approximately twenty spots equally spaced down the filament axis. For subsequent analyses, we assigned an axial coordinate value of 0.0 to the location at the maximum filament diameter and recorded all other diameter measurements relative to this axial position. Independent measurements on a precision reticle verified the precision of the microdensitometer. Measurement of the diameters of glass rods, located in the same plane as the filament, verified the accuracy of the overall photographic system when compared to shadowgraph and direct micrometer measurements. Filament diameters at the position of the laser beam ranged from $0.69 \mathrm{~mm}$ to $2.48 \mathrm{~mm}$, with the largest diameters corresponding to the Separan AP 273 solutions at the high volumetric flow rate and lowest draw wheel speed. Photographs of filaments are reproduced in Talbott (31).

The entire optical system, shown schematically in figure 2, rested upon a massive support table designed to provide a sufficiently vibration-free base for the experiments. The light source was a Spectra-Physics Model $116 \mathrm{He}-\mathrm{Ne}$ laser with a power output of $42 \mathrm{~mW}$ in the $\mathrm{TEM}_{00}$ mode at the wavelength $632.8 \mathrm{~nm}$. A system of baffles around the laser prevented spurious light from entering the system. The beam chopper modulated the light from the laser and generated a reference signal for subsequent use by the lock-in amplifier.

The combination lens L 1-pinhole-lens L 2 expanded the laser beam. The half-wave plate rotated the azimuth of principal polarization of the laser beam to $45^{\circ}$ with respect to the axis of the filament. Two slits, one vertical and one horizontal, limited the beam to $1 \mathrm{~mm}$ by $1 \mathrm{~mm}$ in size. A quarter-wave plate could be inserted preceding the analyzer.

The extending filament was located at the front focal plane of lens L 3 and the mask at its rear focal plane. The function of this arrangement is illustrated in a simplified manner in figure 3. Lens L 3 focuses those rays passing around the filament on the mask but collimates those light rays passing through the filament and directs them to the detector. The operation of this combination corresponds to the principle of the darkfield microscope and a spatial-filtering operation (33). Talbott (31) provides a more detailed analysis of this arrangement including the effects of the limiting aperture at the analyzer, of filament movements, and of rays passing through the filament but blocked by the mask. Included in this analysis is a consideration of the light intensity distribution of the diffraction pattern at the location of the mask. The result of these analyses shows

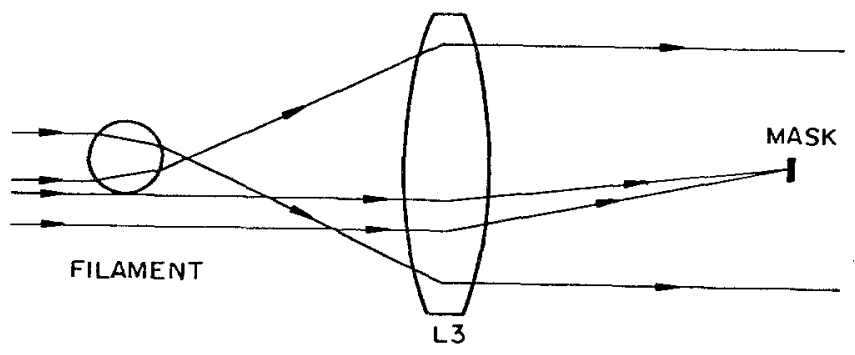

Fig. 3. Simplified action of the mask 
this simplified action of the mask to be valid, introducing no significant errors into the measurement.

The light passed through an interference filter and a ground-glass diffuser to be detected by a 1 P 21 photomultiplier tube in a special housing. A lock-in amplifier accepted the output from the photomultiplier. An ultraviolet-light oscillograph simultaneously recorded the signals from the lock-in amplifier and the nozzle force transducer.

The difficulties associated with light propagation through a circular cross-section gave cause for special attention. Talbott (31) provides the details of the analysis and we give only a brief summary of results here. For the arrangement of components in the experiments, only paraxial rays with respect to the filament reach the detector. Consequently, the filament diameter represents the optical pathlength through the filament with negligible (less than $0.05 \%$ ) error. In addition, the change in polarization caused by interactions with the filament boundaries is less than $0.01 \%$ and likewise negligible. Finally, those paraxial rays blocked by the mask contribute negligible error to the measurement.

The filament acts as an anisotropic cylindrical lens, so that the pair of orthogonally polarized beams emerging from the filament have different beam divergences. For the detection method used, however, this situation introduces no artifact into the measurement (31).

We did not observe significant filament movements or diameter oscillations under the condition for which the data were obtained. The long exposure (one second) photographs presented clear and sharp filament boundaries.

\section{Results and discussion}

\subsection{Extensional flow behavior}

We attempted, as done by Weinberger (32), to relate local stress to the local extension rate at various points along the filament, although this is not strictly necessary for an assessment of the stress-optical behavior. Figures 4 through 8 show these local data for the five solutions. Each set of points represents one combination of volumetric flow rate and draw wheel speed. An increased draw wheel speed displaced each set of points toward the upper right-hand position in each figure.

The correlation of Weinberger $(30,32)$ for his data on a $1 \frac{1}{2} \%$ Separan AP 30 solution in water/ glycerol is superposed on figure 4 for purposes of comparision. (Symbol-experiment identification for figures 4 through 8 is shown in table 2.) The major error in this type of experiment results from uncertainty in the local extension rate which must be calculated by numerical differentiation of diameter data. The dashed lines bracketing Weinberger's correlation show a band of $\pm 15 \%$ in extension rate based upon his error estimates. The current results fall within the same range as those of Weinberger with some degree of overlap.

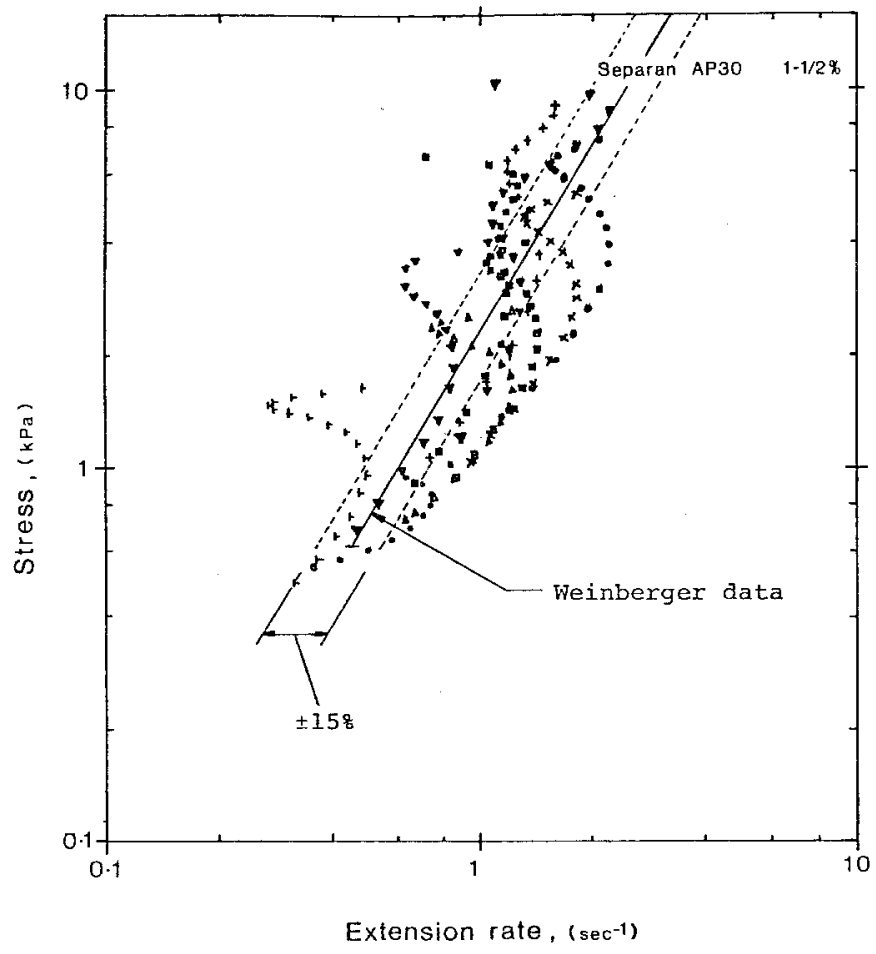

Fig. 4. Local tensile stress - local extension rate plot for $1 \frac{1}{2} \%$ Separan AP 30 solution 

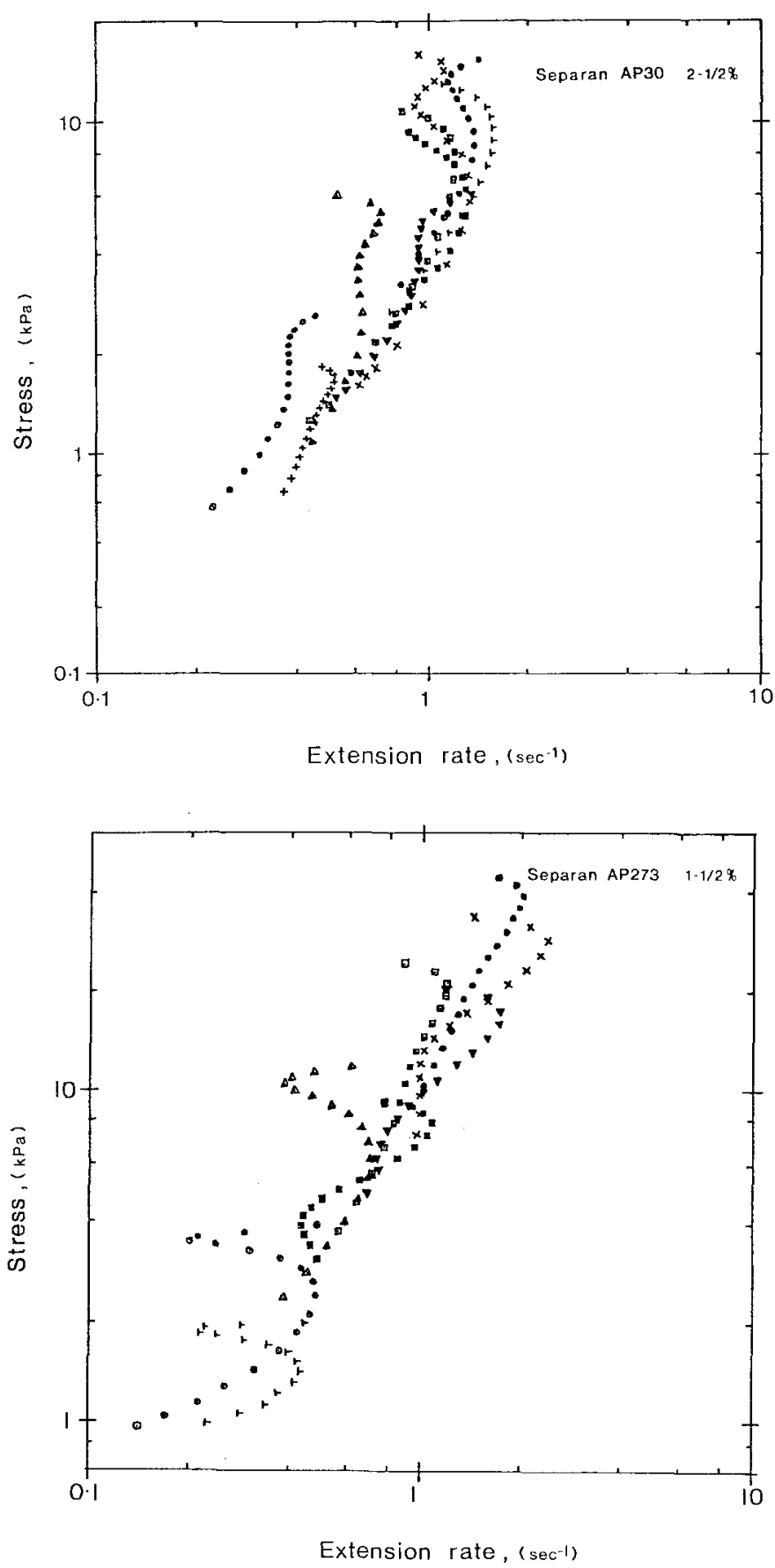

Fig. 5. Local tensile stress - local extension rate plot for $2 \frac{1}{2} \%$ Separan AP 30 solution
Fig. 6. Local tensile stress - local extension rate plot for $1 \frac{1}{2} \%$ Separan AP 273 solution
For the other solutions in this study, shown in figures 5 through 8 , no prior extensional flow studies appear to exist. We see general behavior similar to the $1 \frac{1}{2} \%$ AP 30 solution but with somewhat more data scatter.

The front-lighting arrangement for the filament photographs may cause a slight aberration in the diameter measurements because of light reflected from the filament (34). Any such aberration would greatly amplify variations in the calculated local extension rate after numerical differentiation of the filament profile, and may account for the sigmodial character of the stressstrain rate curves in figures 4 through 8 . Thus, these figures reflect the general difficulty in accurate measurement of the extension rate. 


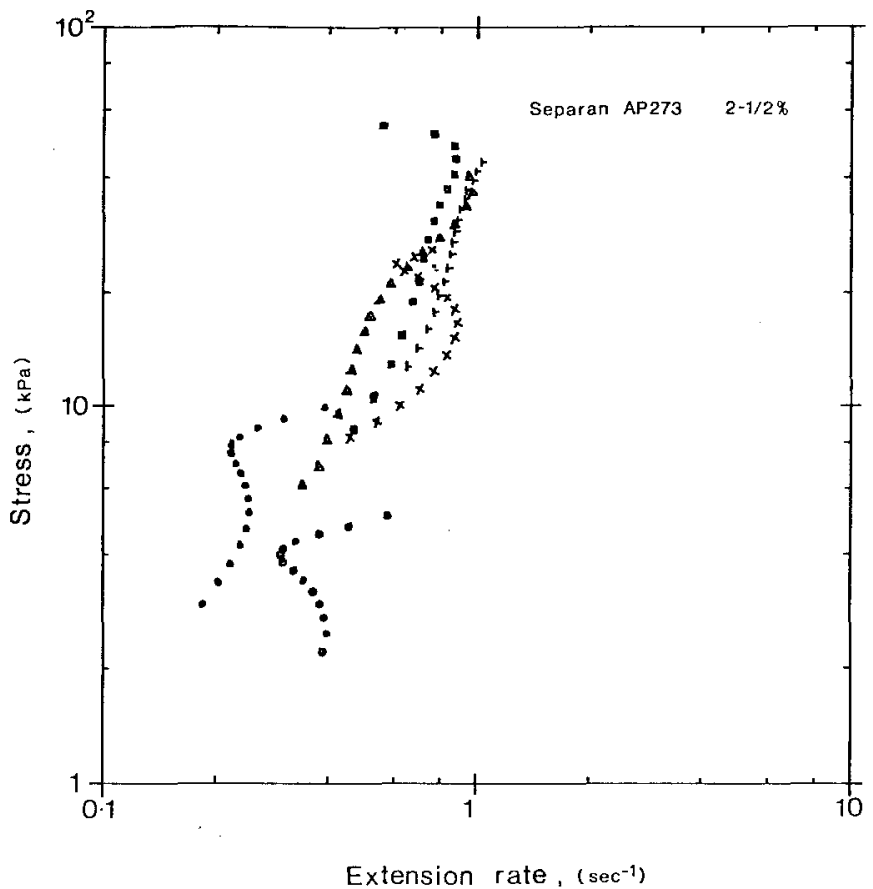

Extension rate, $\left(\mathrm{sec}^{-1}\right)$

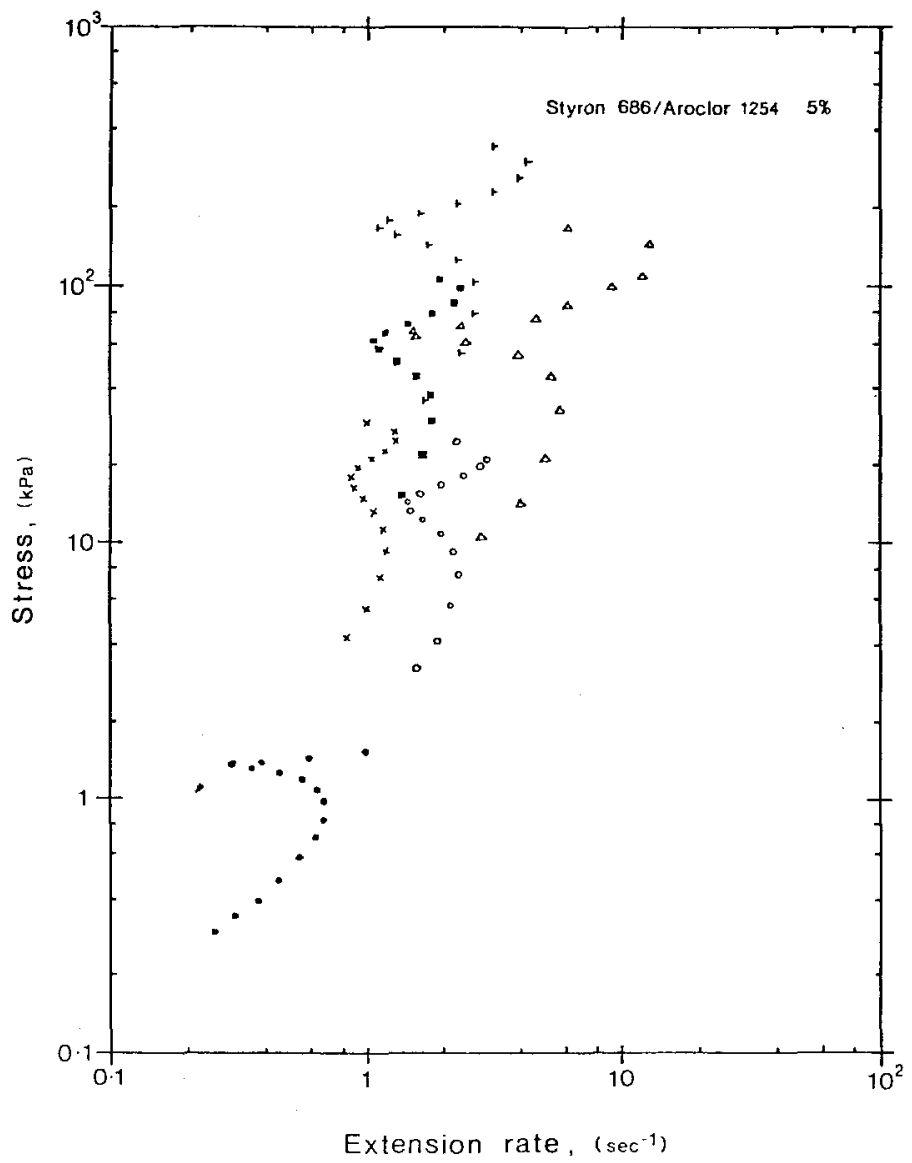

Fig. 7. Local tensile stress - local extension rate plot for $2 \frac{1}{2} \%$ Separan AP 273 solution

Fig. 8. Local tensile stress - local extension rate plot for 5\% Styron 686/ Aroclor 1254 solution 
Table 2. Symbol - experiment identification for local tensile stress - local extension rate plots

\begin{tabular}{|c|c|c|c|}
\hline Figure & Symbol & $\begin{array}{l}\text { Flow rate, } \\
Q \\
\left(\mathrm{~cm}^{3} / \mathrm{sec}\right)\end{array}$ & $\begin{array}{l}\text { Draw wheel } \\
\text { speed } \\
(\mathrm{rad} / \mathrm{sec})\end{array}$ \\
\hline 4 & $\begin{array}{l}\vdash \\
\nabla \\
\square \\
+ \\
\nabla \\
0 \\
\triangle \\
\square \\
x \\
0\end{array}$ & $\begin{array}{l}.0249 \\
.0249 \\
.0249 \\
.0249 \\
.0249 \\
.0744 \\
.0744 \\
.0744 \\
.0744 \\
.0744\end{array}$ & $\begin{array}{l}.0426 \\
.113 \\
.178 \\
.240 \\
.301 \\
.0426 \\
.113 \\
.178 \\
.240 \\
.301\end{array}$ \\
\hline 5 & $\begin{array}{l}0 \\
\triangle \\
\square \\
\times \\
+ \\
\nabla \\
\square \\
\end{array}$ & $\begin{array}{l}.0249 \\
.0249 \\
.0249 \\
.0249 \\
.0744 \\
.0744 \\
.0744 \\
.0744 \\
.0744\end{array}$ & $\begin{array}{l}.0426 \\
.113 \\
.178 \\
.301 \\
.0426 \\
.113 \\
.178 \\
.240 \\
.301\end{array}$ \\
\hline 6 & $\begin{array}{l}0 \\
\triangle \\
\square \\
\vdash \\
\mathbf{0} \\
\times \\
\times \\
0\end{array}$ & $\begin{array}{l}.0249 \\
.0249 \\
.0249 \\
.0744 \\
.0744 \\
.0744 \\
.0744 \\
.0744\end{array}$ & $\begin{array}{l}.0426 \\
.113 \\
.178 \\
.0426 \\
.113 \\
.178 \\
.240 \\
.301\end{array}$ \\
\hline 7 & $\begin{array}{l}0 \\
\triangle \\
0 \\
\times \\
-\end{array}$ & $\begin{array}{l}.0249 \\
.0249 \\
.0249 \\
.0744 \\
.0744 \\
.0744\end{array}$ & $\begin{array}{l}.0426 \\
.113 \\
.178 \\
.0426 \\
.113 \\
.178\end{array}$ \\
\hline 8 & $\begin{array}{l}0 \\
\times \\
\mathbf{a} \\
- \\
0 \\
\Delta\end{array}$ & $\begin{array}{l}.0246 \\
.0246 \\
.0246 \\
.0246 \\
.0404 \\
.0393\end{array}$ & $\begin{array}{l}.0178 \\
.0768 \\
.1275 \\
.1783 \\
.0768 \\
.1275\end{array}$ \\
\hline
\end{tabular}

At any rate, we should not expect a correlation to exist between the local instantaneous stress and the local instantaneous extension rate $(32,35)$ for this materially unsteady flow because of relaxation or memory effects. However, in view of the generality often claimed for the stress-optical relation [1], and on the basis of supporting molecular theories such as that of Lodge (6), such effects should not vitiate the correlation between stress and birefringence. Indeed, the shear flow results of Janeschitz-
Kriegl and coworkers $(14,15)$ tend to support the validity of the linear law [1] in transient experiments which, in view of the results to be presented below, may be a consequence of the relatively low shear rates they studied.

\subsection{Stress-optical properties of the solutions in extensional flow}

Figure 9 shows our results for Separan AP 30 solutions. The straight line visually drawn through the data points has no special significance other than to indicate the trend in the data. Vertical bars represent the spread in birefringence

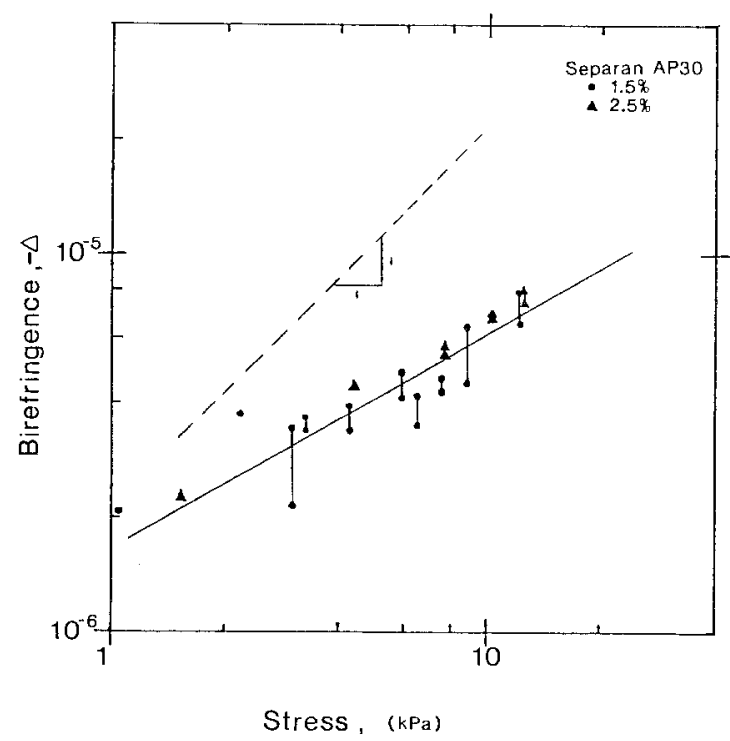

Fig. 9. Dependence of local birefringence upon local tensile stress for Separan AP 30 solutions

values from replicated data points. Within the limits of experimental error, we note no effect of concentration. The dashed line at the upper left is a line of unit slope representing the classical linear stress-optical relation. Our results indicate definite non-linear behavior, even at the nominally low deformation rates involved $\left(\dot{\varepsilon}<10 \mathrm{sec}^{-1}\right)$.

We show our results for the Separan AP 273 solutions in figure 10 , and notice behavior similar to Separan AP 30. Again, we detect no effect of concentration and we observe definite non-linear stress-optical behavior.

Finally, we present our results for the Styron 686/Aroclor 1254 solution in figure 11 which again show non-linear behavior. The negative birefringence observed here implies that the 
net polarizability of the solution perpendicular to the axis of extension of the solution is greater than that parallel to the axis. Since the polymer molecules are presumably extended parallel to this direction, this result was anticipated from the fact that all of the polymers in this study are derived from monomers having rather large or polar side groups.

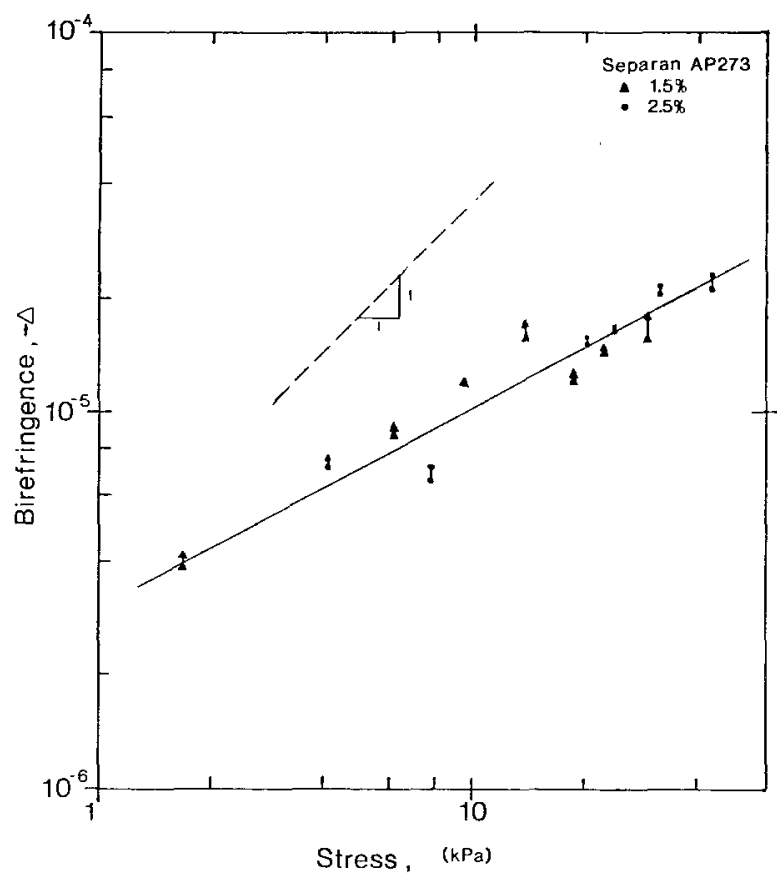

Fig. 10. Dependence of local birefringence upon local tensile stress for Separan AP 273 solutions
Whereas the stress-optical properties of the polyacrylamide solutions do not appear to have been studied before, either in shear flow or in extensional flow, the same is not true of polystyrenes. We summarize the shear experiments of Philippoff $(11,12)$, on two distinct polystyrenes in Aroclor 1248, by the straight line in figure 12 (determined from his stress-optical coefficient for the shear rate-shear stress range of his studies) and place on this figure our results (see fig. 11) for comparison.

Munk and Peterlin (36), in studies on four different polystyrenes in Aroclors 1248 and 1254 , demonstrated that neither the type of polystyrene nor the type of Aroclor significantly altered the stress-optical coefficient for these solutions. Philippoff (11), furthermore, showed that contributions to the observed birefringence by the solvent itself could be neglected for solutions of this high concentration. Therefore, we suggest that the differences seen in figure 12 are attributable only to the effect of these different flow fields upon the molecules in solution. Philippoff attained the highest stress levels in his studies at shear rates of nearly $1000 \mathrm{sec}^{-1}$, three orders of magnitude higher than the lowest extension rate (corresponding to the lowest stress level) for the flow results of our study. In addition, the birefringence observed here is higher than that observed by Philippoff for comparable solutions. Thus, in extensional flow the birefringence and stress reach com-

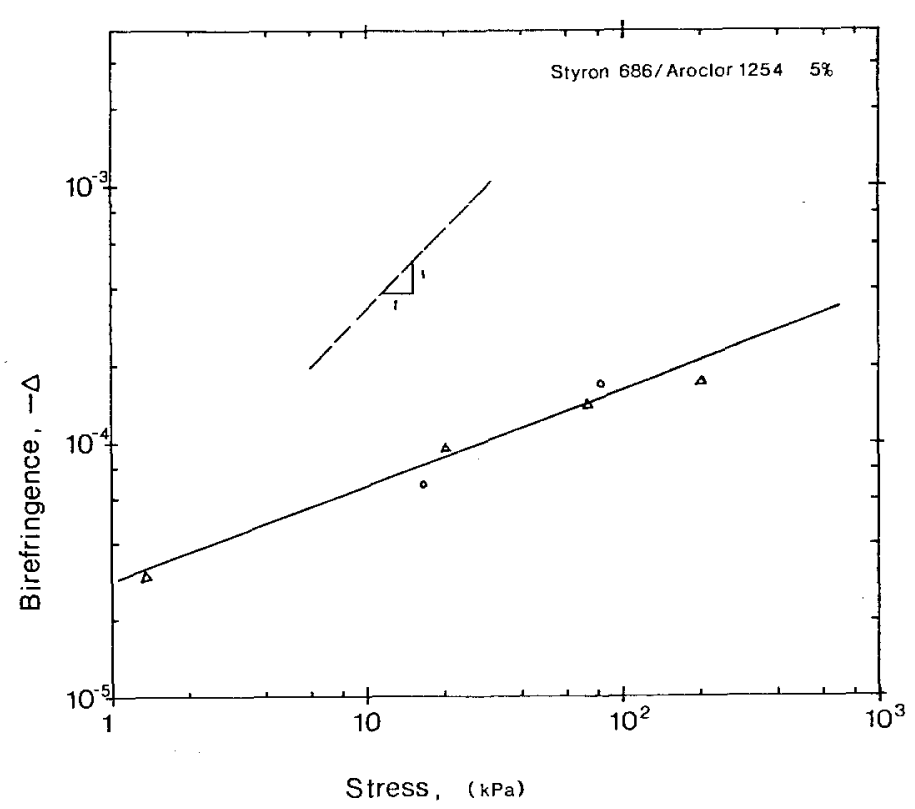

Fig. 11. Dependence of local birefringence upon local tensile stress for 5\% Styron 686/Aroclor 1254 solution 
parable values at much lower deformation rates. Conversely, at comparable deformation rates, both the birefringence and stress are greater in extension than in shear and the relation between the two is apparently non-linear, with the stress increasing more rapidly than the birefringence.

It is of further interest to compare the experimental results of this study with two widely contrasting microrheological theories of polymer solution behavior. As pointed out above, the elastic-liquid or affine network theory $(37,38)$, illustrated qualitatively in figure $13 \mathrm{a}$, predicts a linear stress-optical relation. On the other hand, dilute, rigid-particle theories of suspension or solution rheology $(39,40)$ predict limiting linear behavior at low stress levels and, for particles of large aspect ratios, a limiting saturation of the birefringence at high stress levels corresponding to complete particle alignment. This behavior is qualitatively illustrated in figure $13 \mathrm{~b}$.
Comparison of figures 9 through 12 with figure 13 shows that the linear relation predicted by network theory is not supported by the results of these experiments, while the rigid particle theories seem to qualitatively indicate the trend of our data and the results of Matsumoto and Bogue (28) at high stress levels.

This qualitative agreement does not, of course, imply the appropriateness or validity of rigid particle theories as representations of polymer melts and concentrated solutions. However, these theories do involve the concept of limiting molecular orientation as reflected by birefringence saturation and our experimental results suggest that a relatively high degree of molecular orientation in extension is achieved. This concurs with the expectation of Matsumoto and Bogue (28) of a smaller increase in molecular alignment than in stress for flows in which an appreciable degree of molecular alignment al-
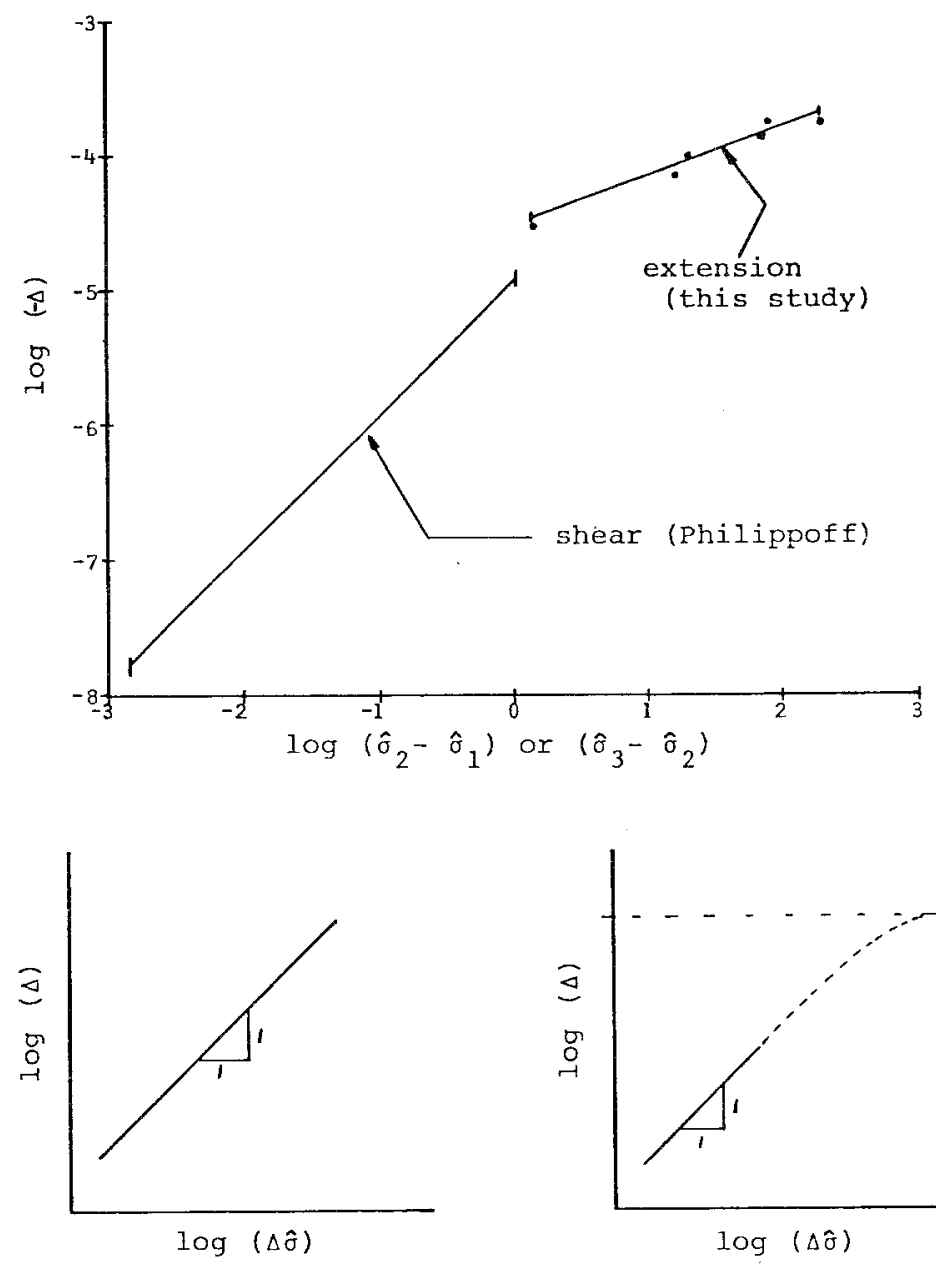

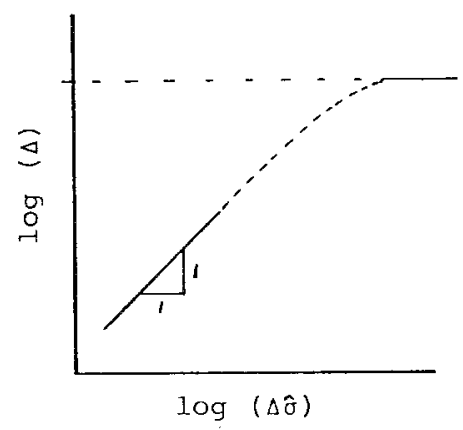

Fig. 12. Comparison of shear and extensional birefringence results for Polystyrene/Aroclor solutions
Fig. 13. Stress-optical relations predicted by network theory and rigid particle theory in both shear and extension. (a) Network theory, (b) rigid particle theory 
ready exists, so that linear stress-optical relations no longer obtain.

In this regard, figure 12 suggests that shear flow data such as that of Philippoff $(11,12)$ and extensional flow data are useful complements.

\section{Conclusion and implications}

Our on-line measurements of birefringence in filaments of polymer solutions lend much support to the concept that extensional motions produce pronounced orientation effects. A quantitative measure of significant molecular orientation is manifest in the non-linear stress-optical behavior, in which flow-induced stress increases more rapidly than birefringence. Furthermore, our observed extensional flow birefringence is nearly two orders of magnitude greater than that for shear, at comparable deformation rates, for the polystyrene/Aroclor solution.

This implied molecular ordering in extensional flow seems intuitively correct in view of numerous other facts. For example, extension has been utilized by the fiber industry in drawspin production processes for partially-oriented yarn and, in addition, extensional flow is known to cause flow-induced crystallization - see, for example, reference (41) - more readily than shear flow.

We feel our results cast further doubt on the universality of the linear stress-optical relation, which is to a large extent based on results from shearing flows and justified by appeal to special molecular theories. On the other hand, we cannot at this time reconcile our findings with the agreement between linear theory obtained in recent on-line measurement of extensional biregringence in polymer melts $(27,28)$, and we feel that much more work in this area is to be highly recommended.

\section{Acknowledgment}

This work was done at the University of Michigan with partial support from National Science Foundation Grant GK 38303 which we gratefully acknowledge. The authors are also indebted to Professor R. L. Curl, for a number of helpful ideas relating to the experimental measurements, and to Professor G.S.Y. Yeh, for his active and sympathetic interest in the progress of the work. Finally, we wish to thank Dr's. W. Brasie and L.H. Tung and associates, of the Dow Chemical Company, Midland, Michigan, for providing wellcharacterized samples of polystyrene.

\section{Summary}

The stress and birefringence in liquid filaments undergoing an extensional flow were measured in an isothermal filament-spinning apparatus. In conjunction with these measurements, an experimental arrangement is described which, as subsequent analyses show, overcomes the difficulties associated with the measurement of birefringence in this geometry.

The fluids studied were $1 \frac{1}{2} \%$ and $2 \frac{1}{2} \%$ solutions of two polyacrylamides (Separan AP 30 and Separan AP 273) in a 1:1 water/glycerol mixture and a 5\% solution of polystyrene (Styron 686) in Aroclor 1254.

For all solutions studied, the measured birefringence exhibited a non-linear relation to the principal stress difference. In addition, for the polystyrene/Aroclor solution, the birefringence measured in extension was nearly two orders of magnitude higher than the birefringence in shear at comparable deformation rates. These observations imply a greater degree of molecular orientation is produced by the extensional flow than the shear flow and suggest that birefringence data obtained in shear and extension are useful complements to depict a wider range of stress-optical behavior.

\section{Sommaire}

On a mesuré l'éffet Maxwell, c'est-à-dire la biréfringence en fonction de la tension, dans des filaments liquides de solution polymérique soumise a un écoulement extensionel. Au moyen d'un appareil spécial, on a réussi a resoudre les problèmes d'optique provenant de cette configuration expérimentale. Avec cet appareil, on a étudié plusieurs solutions aqueuses des polyacrylamides en glycérine aussi bien q'une solution du polystyrene en Arochlor 1254 (polychlor biphenyl).

Pour tous les solutions étudiées, la biréfringence montre une dépendance non-linéaire sur la tension principale. En plus, la biréfringence du polystyrene en extension est à peu pres cent fois plus grande que sa biréfringence en cisaillement, à des taux de déformation pareils. Ces observations supportent l'idée que l'extension simple est bien plus puissante pour l'orientation macromoléculaire que le cisaillement. Donc les deux espèces de biréfringence pourront peut-être servir comme mesures complémentaires de l'éffet Maxwell.

\section{References}

1) Wales, J. L. S., The Application of Flow Birefringence to Rheological Studies of Polymer Melts, Delft University Press (1976).

2) Rivlin, R. S., G. F. Smith, Z. angew. Math. Physics 22, 325 (1971); see also G. F. Smith, R. S. Rivlin, Z. angew. Math. Physics 21, 101 (1970).

3) Coleman, B. D., E. H. Dill, R. A. Toupin, Arch. Rat. Mech. Anal. 39, 358 (1970).

4) Philippoff, W., F. H. Gaskins, J. G. Brodnyan, J. Appl. Physics 28, 1118 (1957).

5) Brodnyan, J. G., F. H. Gaskins, W. Philippoff,

E. G. Lendrat, Trans. Soc. Rheology 2, 285 (1958).

6) Lodge, A. S., Nature 176, 838 (1955).

7) Philippoff, W., J. Appl. Physics 27, 984 (1956).

8) Brodnyan, J. G., F. H. Gaskins, W. Philippoff, Trans. Soc. Rheology 1, 109 (1957). 
9) Wayland, H., J. Polymer Sci.: Part C, Polymer Symposia, No. 5, 11 (1964).

10) Philippoff, $W$., Trans. Soc. Rheology 4, 159 (1960).

11) Philippoff, W., in: E. H. Lee (ed.), Proc. IV Intern. Congr. Rheology Providence 1963, Vol. 2, pp. 343-372, Interscience Publ. (New York 1965).

12) Philippoff, W., R. A. Stratton, in: S. Onogi (ed.), Proc. V Intern. Congr. Rheology Kyoto 1968, Vol. 4, pp. 13-23, University Park Press (Baltimore-Manchester 1970).

13) Harris, $J$., Rheol. Acta 9, 467 (1970).

14) Gortemaker, F. H., M. G. Hansen, B. deCindio, H. M. Laun, H. Janeschitz-Kriegl, Rheol. Acta 15, 256 (1976).

15) Gortemaker, F. H., H. Janeschitz-Kriegl, K. te Nijenhuis, Rheol. Acta 15, 487 (1976).

16) Wales, J. L. S., W. Philippoff, Rheol. Acta 12, 25 (1973).

17) Ziabicki, A., K. Kedzierska, J. Appl. Polymer Sci. 2, 14 (1959).

18) Ziabicki, A., K. Kedzierska, J. Appl. Polymer Sci. 6, 111 (1962).

19) Ziabicki, A., K. Kedzierska, J. Appl. Polymer Sci. 6, 361 (1962).

20) Thompson, A. B., in: J.W.S. Hearle, R. H. Peters (eds.), Fibre Structure, Chapter 14, pp. 480-523, Textile Institute-Butterworths (Manchester-London 1963).

21) Chappel, F. P., M. P. Culpin, R. G. Gosden, T. C. Tranter, J. Appl. Chem. 14, 12 (1964).

22) Katayama, K., T. Amano, K. Nakamura, Koll.-Z. Z. Polym. 226, 125 (1968).

23) Hamana, I., M. Matsui, S. Kato, Melliand Textilber. 50, 382 (1969).

24) Ishibashi, T., K. Aoki, T. Ishii, J. Appl. Polymer Sci. 14, 1597 (1970).

25) Dees, J. R., J. E. Spruiell, J. Appl. Polymer Sci. 18, 1053 (1974).

26) Ishibashi, T., J. Furukawa, J. Appl. Polymer Sci. 20, 1421 (1976).
27) Oda, K., J. L. White, E. S. Clark, Polym. Eng. Sci. 18, 53 (1978).

28) Matsumoto, T., D. C. Bogue, J. Polymer Sci., Polymer Phys. Ed. 15, 1663 (1977).

29) Peterlin, A., in: M. Van Dyke, W. G. Vincenti, J. $V$. Wehausen (eds.), Annual Reviews of Fluid Mechanics, Vol. 8, pp. 35-55, Annual Reviews (Palo Alto 1976).

30) Weinberger, C. B., Ph. D. thesis, The University of Michigan (Ann Arbor 1970).

31) Talbott, W. H., Ph. D. thesis, The University of Michigan (Ann Arbor 1978).

32) Weinberger, C. B., J. D. Goddard, Int. J. Multiphase Flow 1, 465 (1974).

33) Goodman, J.W., Introduction to Fourier Optics, McGraw-Hill (New York 1968).

34) Weinberger, C. B., personal communication.

35) Baid, K. M., A. B. Metzner, Trans. Soc. Rheology 21, 237 (1977).

36) Munk, P., A. Peterlin, Rheol. Acta 9, 294 (1970).

37) Lodge, A. S., Kolloid Z. 171, 46 (1960).

38) Lodge, A. S., Elastic Liquids, Academic Press (New York 1964).

39) Brenner, $H$., Int. J. Multiphase Flow 1, 195 (1974).

40) Leal, L. G., E. J. Hinch, Rheol. Acta 11, 190 (1972).

41) Mackley, M. R., A. Keller, Polymer 14, 16 (1973).

Authors' addresses:

Dr. W. H. Talbott

Department of Chemical Engineering

Clemson University

Clemson, S.C. 29631 (USA)

Dr. J. D. Goddard

Department of Chemical Engineering

University of Southern California

University Park

Los Angeles, C.A. 90007 (USA) 